\title{
Behavioral Intention to Use IoT Technology in Healthcare Settings
}

\author{
Meshari Alanazi \\ Department of Computer Science and Information \\ Technology, La Trobe University, \\ Melbourne, Australia \\ m.alanazi@latrobe.edu.au
}

\author{
Ben Soh \\ Department of Computer Science and Information \\ Technology, La Trobe University, \\ Melbourne, Australia \\ m.soh@latrobe.edu.au
}

\begin{abstract}
Rapid scaling of using the Internet of Things (IoT) technology has been seen recently in numerous applications in healthcare to deliver proper services. This was motivated by the declining size and cost of the employed IoT devices. Developing such technology has been well investigated in the literature; however, few studies have explored the factors influencing its adaptation in the healthcare setting. In this study, we investigate the core factors that influence the acceptance of using IoT for Healthcare Purposes in the Kingdom of Saudi Arabia (KSA). Accordingly, a theoretical framework, based on the Technology Acceptance Model (TAM), was developed and tested empirically. The modified model added variables that provide a better explanation of the acceptance of healthcare technology. To ground our conceptual idea, a survey was designed and performed on 407 patients (207 males, 200 females). The Partial Least Square Structural Equation Modeling (SEM) technique was applied to analyze the effect of eight hypothesized predicting constructs on the collected data. Results revealed that cost, privacy concerns, and perceived usefulness were the most significant predictors of behavioral intention to use. However, attitude and perceived connectedness were found to be irrelevant in predicting the intention to use IoT. Ultimately, results found that there is no correlation between gender and behavioral intention.
\end{abstract}

Keywords-Internet of Things; healthcare; technology acceptance model (TAM); structural equation model

\section{INTRODUCTION}

Internet of Things (IoT) technology is a system of interrelated smart devices (computers, sensors, etc.) that are connected to the Internet to develop new capabilities and services [1]. These services aim to improve system performance and quality of life, e.g. in healthcare, communication, education, etc. [2]. IoT has many advantages to offer, for example, the Internet of Medical Things (or the internet of healthy things). Internet of Medical Things is an application of IoT for medical and health-related purposes [3], where IoT devices (devices for monitoring blood pressure, heart rate, or specialized implants, such as pacemakers, or advanced hearing aids) are used to enable remote health monitoring process [4]. This process can significantly improve the quality of life for patients, especially for chronic diseases, as they can be monitored in non-clinical environments such as their home. While IoT delivers an impressive set of benefits, concern exists over the extent of IoT technology use by patients. In other words, as any new technology proposed, it has the potential to enhance the provided services and achieve its goals when intended users understand it. But, if the technology meets resistance to use or acceptance, it will be underutilized or completely abandoned. The lack of user acceptance has been long identified as an obstacle to the success of new technologies [5]. Therefore, it has become crucial for practitioners and decision-makers to better understand the factors that influence the adoption of IoT, since it is considered an essential step toward the development of a successful healthcare system [6].

Many models and theories have been proposed to examine and predict the acceptance of new technologies. One might consider the Technology Acceptance Model (TAM) [7], the Unified Theory of Acceptance and Use Technology [8] and the Theory of Reasoned Action [9]. The TAM model is employed in this study to understand and estimate the users' adoption behaviors toward modern technologies [7]. This is due to the fact that TAM is the most common and cited model in the IS research [10] and healthcare domain $[11,12]$, because of its adequate explanatory power and parsimonious structure [13]. Being said that, TAM has not been widely tested in developing countries [14]. Consequently, the author of [15] emphasizes on the importance of examining TAM in different cultures to ensure adequate reliability and validity. Moreover, TAM does not serve equally across cultures, and the inconsistency in previous studies' results highlight the importance of conducting this research in the KSA context $[16,17]$. KSA remains relatively unexplored in terms of technology acceptance, while investment in healthcare system technology is promised for future projects. Hence, this study aims to examine the individual patients' perceptions towards the adoption of IoT technology in KSA by applying the TAM model.

\section{RESEARCH MODEL}

Our purpose is to study the acceptance of using IoT technology in the healthcare sector in KSA from a conceptual viewpoint. We propose a theoretical model that extends TAM with its original four factors, Perceived Usefulness (PU), 
Perceived Ease of Use (PEO), Attitude (ATT), and Behavioral Intention to Use (BI), including four new external factors: Connectedness (PCO), Convenience (PCV), Privacy (PPR), and Cost (PC) (Figure 1).

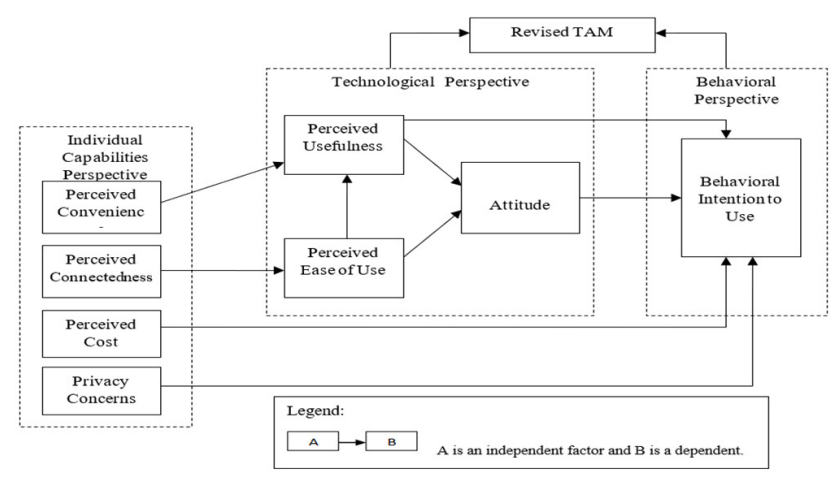

Fig. 1. The proposed research model

A survey instrument was developed and distributed to 450 patients in order to test the research model [37]. Collected data were analyzed using the Structural Equation Model, based on the Partial Least Square approach. This method shows relationships and the quality of connections among the factors in the proposed model. In particular, we want to assess the influence of the four original factors on the intention of using IoT, by specifying the relationships between them and among the new factors that subsequently influence the usage intention. Accordingly, a set of hypotheses were set to determine if there is a correlation between these factors. Those hypotheses are listed in Table I.

TABLE I. RESEARCH HYPOTHESES

\begin{tabular}{|c|c|}
\hline \multicolumn{2}{|c|}{ Hypotheses } \\
\hline H1 & Perceived Convenience has direct effect on Perceived Usefulness \\
\hline H2 & Perceived Connectedness has direct effect on Perceived Ease of Use \\
\hline H3 & Perceived Cost has direct effect on Behavioral Intention to Use \\
\hline H4 & Privacy Concern has direct effect on Behavioral Intention to Use \\
\hline H5 & Perceived Ease of Use has direct effect on Perceived Usefulness \\
\hline H6 & Perceived Usefulness has direct effect on Attitude \\
\hline H7 & Perceived Ease of Use has direct effect on Attitude \\
\hline H8 & Perceived Usefulness has direct effect on Behavioral Intention to Use \\
\hline H9 & Attitude has direct effect on Behavioral Intention to Use \\
\hline
\end{tabular}

Before conducting the survey, two independent experts reviewed the instrument for ensuring its validity and relevance. The instrument was structured in two parts. The first part contained sociodemographic questions and a basic question on IoT awareness, which was "Do you have basic knowledge of IoT?", with response options ranging between "General Idea", "Good Idea," and "Already using some Services". The second part contained questions that measured the respondents' intention to use IoT for healthcare, utilizing a five-point Likert scale (from 1="strongly disagree" to 5="strongly agree"). The interval scale was used because it allows specific mathematical operations on the data collected from respondents. In order to ensure that the survey will be distributed over a sufficient number of participants so as to generalize our model, the Cochran's Sample Size Formula was utilized to calculate the minimum sample size, as it is proven reliable for use in large populations [18]. The current study targeted adult patients and according to the KSA General Authority for Statistics data in 2016 the proportion of the adult population of Saudi Arabia is $75.2 \%$, which equals to $23,870,215$ persons. The Cochran's Sample Size Formula [18] is given as:

$$
\text { no }=\frac{z^{2}(p * q)}{e^{2}}
$$

where $e$ is the desired level of perception (error margin), $p$ is the estimated proportion of the population which has the attribute in question, $q=1-p$ and $z$-value is found in the $\mathrm{Z}$ table. In this study, we desired $95 \%$ confidence level, $\mathrm{p}=0.05$ and at least $5 \%$. A $95 \%$ confidence level gives us $z$-value of 1.96 , so, based on (1), the minimum sample size is 385 . The instrument was distributed to 450 patients in KSA hospitals and 426 responses were obtained, out of which 19 were excluded because of missing values. Hence, data from 407 respondents were processed for the final analysis.

\section{TESTING RESUlts}

\section{A. Respondents Demographic Statistics}

Descriptive statistics showing the respondents demography can be seen in Table II. There were 207 male and 200 female participants, where $27.5 \%$ of them were 55 years old or older, $21.1 \%$ were $45-54$ years old, $20.1 \%$ were $35-44$ years old, $16.5 \%$ were $25-34$ years old, and the remainder $14.7 \%$ were $18-24$ years old. $48.2 \%$ of our sample had basic knowledge of IoT, while the rest either had a good idea or already used some IoT services. Regarding the income, $23.8 \%$ registered high income with more than $190 \mathrm{~K} \mathrm{SR}, 40.3 \%$ had income $120 \mathrm{~K}$ 190K SR, $20.4 \%$ had income $48 \mathrm{~K}-120 \mathrm{~K} \mathrm{SR}$, and $15.5 \%$ had less income than $48 \mathrm{~K}$ SR.

TABLE II. DEMOGRAPHIC INFORMATION OF THE RESPONDENTS

\begin{tabular}{|c|c|c|c|}
\hline \multicolumn{2}{|c|}{ Characteristics } & Frequency & Percent \% \\
\hline \multirow{4}{*}{ Age } & $18-24$ & 60 & 14.7 \\
\cline { 2 - 4 } & $25-34$ & 67 & 16.5 \\
\cline { 2 - 4 } & $35-44$ & 82 & 20.1 \\
\cline { 2 - 4 } & $45-54$ & 86 & 21.1 \\
\cline { 2 - 4 } & 55 or older & 112 & 27.5 \\
\hline \multirow{4}{*}{ Gender } & Total & $\mathbf{4 0 7}$ & $\mathbf{1 0 0 . 0}$ \\
\cline { 2 - 4 } & Male & 207 & 50.9 \\
\cline { 2 - 4 } & Female & 200 & 49.1 \\
\hline \multirow{4}{*}{$\begin{array}{c}\text { Income } \\
\text { (SR) }\end{array}$} & Total & $\mathbf{4 0 7}$ & $\mathbf{1 0 0 . 0}$ \\
\cline { 2 - 4 } & <48K & 63 & 15.5 \\
\cline { 2 - 4 } & 48K-120K & 83 & 20.4 \\
\cline { 2 - 4 } & $120 \mathrm{~K}-190 \mathrm{~K}$ & 164 & 40.3 \\
\hline \multirow{4}{*}{$\begin{array}{c}\text { Basic } \\
\text { knowledge } \\
\text { of IoT }\end{array}$} & Total & 97 & 23.8 \\
\cline { 2 - 4 } & General idea & $\mathbf{4 0 7}$ & $\mathbf{1 0 0 . 0}$ \\
\cline { 2 - 4 } & Good idea & 196 & 48.2 \\
\cline { 2 - 4 } & Already using IoT & 67 & 35.4 \\
\hline \multirow{2}{*}{ Total } & $\mathbf{4 0 7}$ & $\mathbf{1 0 0 . 0}$ \\
\hline
\end{tabular}

\section{B. Normality Testing}

Skewness and kurtosis have been calculated using the SPSS platform to test the normality of the used data set. Normality tests are used to determine whether a dataset has normal distribution. In this study, skewness was used to measure the asymmetry of the probability distribution of a random variable about its mean, while Kurtosis was utilized to tell the height 
and sharpness of the central peak, relative to the standard bell curve. Skewness and kurtosis values can be positive or negative or even undefined. If skewness value is between -0.5 and 0.5 , and if kurtosis value is between -2 and +2 , they are acceptable and prove normal univariate distribution [19]. As shown in Table III, all factors have obtained values in the acceptable range, which reflects a high degree of normality.

TABLE III. NORMALITY OF THE DATASET

\begin{tabular}{|c|c|c|c|}
\hline \multicolumn{2}{|l|}{ Factor } & Skewness & Kurtosis \\
\hline \multirow{3}{*}{$\begin{array}{l}\text { Behavioural Intention to Use } \\
\text { (BI) }\end{array}$} & BI1 & 0.453 & -0.802 \\
\hline & BI2 & 0.387 & -1.128 \\
\hline & BI3 & 0.197 & -1.133 \\
\hline \multirow{6}{*}{$\begin{array}{l}\text { Perceived Usefulness } \\
\text { (PU) }\end{array}$} & PU1 & -0.092 & -0.084 \\
\hline & PU2 & 0.136 & -0.037 \\
\hline & PU3 & 0.089 & -0.133 \\
\hline & PU4 & 0.042 & -0.348 \\
\hline & PU5 & 0.075 & -0.058 \\
\hline & PU6 & -0.030 & -0.201 \\
\hline \multirow{6}{*}{$\begin{array}{l}\text { Perceived Ease of Use } \\
\text { (PEO) }\end{array}$} & PEO1 & 0.017 & -0.705 \\
\hline & PEO2 & 0.099 & -0.592 \\
\hline & PEO3 & 0.141 & -0.581 \\
\hline & PEO4 & 0.244 & -0.824 \\
\hline & PEO5 & 0.140 & -0.442 \\
\hline & PEO6 & 0.087 & -0.605 \\
\hline \multirow{3}{*}{$\begin{array}{l}\text { Attitude } \\
\text { (ATT) }\end{array}$} & ATT1 & -0.062 & -0.535 \\
\hline & ATT2 & -0.132 & -0.440 \\
\hline & ATT3 & 0.030 & -0.864 \\
\hline \multirow{3}{*}{$\begin{array}{l}\text { Perceived Connectedness } \\
\text { (PCO) }\end{array}$} & PCO1 & 0.328 & -0.114 \\
\hline & PCO2 & 0.237 & 0.044 \\
\hline & PCO3 & 0.240 & -0.268 \\
\hline \multirow{3}{*}{$\begin{array}{l}\text { Perceived Cost } \\
\text { (PC) }\end{array}$} & PC1 & -0.262 & -1.083 \\
\hline & PC2 & -1.035 & -0.272 \\
\hline & PC3 & -0.533 & -1.092 \\
\hline \multirow{3}{*}{$\begin{array}{l}\text { Privacy Concerns } \\
\text { (PPR) }\end{array}$} & PPR1 & 0.462 & -0.842 \\
\hline & PPR2 & 0.578 & -0.825 \\
\hline & PPR3 & 0.349 & -1.174 \\
\hline \multirow{3}{*}{$\begin{array}{l}\text { Perceived Convenience } \\
\text { (PCV) }\end{array}$} & PCV1 & 0.474 & -0.417 \\
\hline & PCV2 & 0.500 & -0.482 \\
\hline & PCV3 & 0.477 & -0.288 \\
\hline
\end{tabular}

\section{Validity Testing}

Cronbach's alpha is calculated to measure the internal consistency for the reliability of the used questionnaire, measuring how a set of items is closely related as a group. Table IV presents all the factors that are used, where the value of Cronbach's alpha obtained is greater than 0.7 , which reflects a high degree of internal reliability [20].

TABLE IV. INSTRUMENT'S INTERNAL CONSISTENCY

\begin{tabular}{|c|c|c|}
\hline Factor & No. of Questions & Cronbach's Alpha \\
\hline BI & 3 & 0.894 \\
\hline PU & 6 & 0.929 \\
\hline PEO & 6 & 0.874 \\
\hline ATT & 3 & 0.939 \\
\hline PCO & 3 & 0.863 \\
\hline PC & 3 & 0.883 \\
\hline PPR & 3 & 0.783 \\
\hline PCV & 3 & 0.875 \\
\hline
\end{tabular}

\section{Convergent Validity}

In order to measure the convergent validity, the average variance extracted (AVE) and the composite reliability have been calculated for every factor, as shown in Tables V and VI. The corresponding factor loading for every construct exceeds the threshold value of 0.60 , which is a minimum requirement criterion for the convergent validity test to pass [21]. Also, for every construct, the value obtained for AVE is higher than the recommended level of 0.5 [22].

TABLE V. FACTOR LOADING

\begin{tabular}{|c|c|c|c|c|c|c|c|c|}
\hline & $\mathbf{P U}$ & PEO & ATT & PCV & PCO & PC & PPR & BI \\
\hline \multirow{6}{*}{ PU } & 0.793 & & & & & & & \\
\hline & 0.761 & & & & & & & \\
\hline & 0.820 & & & & & & & \\
\hline & 0.829 & & & & & & & \\
\hline & 0.898 & & & & & & & \\
\hline & 0.863 & & & & & & & \\
\hline \multirow{6}{*}{ PEO } & & 0.701 & & & & & & \\
\hline & & 0.745 & & & & & & \\
\hline & & 0.729 & & & & & & \\
\hline & & 0.722 & & & & & & \\
\hline & & 0.738 & & & & & & \\
\hline & & 0.761 & & & & & & \\
\hline \multirow{3}{*}{ ATT } & & & 0.912 & & & & & \\
\hline & & & 0.984 & & & & & \\
\hline & & & 0.859 & & & & & \\
\hline \multirow{3}{*}{ PCV } & & & & 0.816 & & & & \\
\hline & & & & 0.829 & & & & \\
\hline & & & & 0.866 & & & & \\
\hline \multirow{3}{*}{ PCO } & & & & & 0.806 & & & \\
\hline & & & & & 0.955 & & & \\
\hline & & & & & 0.718 & & & \\
\hline \multirow{3}{*}{ PC } & & & & & & 0.802 & & \\
\hline & & & & & & 0.867 & & \\
\hline & & & & & & 0.869 & & \\
\hline \multirow{3}{*}{ PPR } & & & & & & & 0.771 & \\
\hline & & & & & & & 0.816 & \\
\hline & & & & & & & 0.650 & \\
\hline \multirow{3}{*}{ BI } & & & & & & & & 0.822 \\
\hline & & & & & & & & 0.861 \\
\hline & & & & & & & & 0.887 \\
\hline
\end{tabular}

TABLE VI. CONVERGENT VALIDITY

\begin{tabular}{|c|c|c|c|}
\hline Factor & No. of questions & $\begin{array}{l}\text { Average variance } \\
\text { extracted }(>0.50)\end{array}$ & $\begin{array}{c}\text { Composite reliability } \\
(>0.70)\end{array}$ \\
\hline BI & 3 & 0.734591 & 0.892418 \\
\hline $\mathbf{P U}$ & 6 & 0.686464 & 0.929071 \\
\hline PEO & 6 & 0.537153 & 0.874351 \\
\hline ATT & 3 & 0.84596 & 0.942609 \\
\hline PCO & 3 & 0.692395 & 0.869442 \\
\hline PC & 3 & 0.716685 & 0.883432 \\
\hline PPR & 3 & 0.560932 & 0.791627 \\
\hline$\overline{P C V}$ & 3 & 0.701018 & 0.87546 \\
\hline
\end{tabular}

\section{E. Discriminant/Divergent Validity}

The discriminant/divergent validity was also tested. Discriminant validity refers to the extent to which factors are distinct and uncorrelated. The rule is that variables should relate more strongly to their factor than to any other factor. The test was calculated using SPSS, and the result is shown in Table VII. When examining discriminant validity, the square root of the AVE for each construct should be greater than the correlational values between any two constructs. This is precisely our case: all diagonal elements have a higher correlation level between any two specific factors. Thus, the discriminant validity test is sufficed for our model. 


\section{HYPOTHESES RESULTS}

AMOS 23.0 has been used to test the proposed model and the corresponding hypotheses. Figure 2 and Table VII present the results of the research model. As shown, p-value is used to determine the significance of the results. In other words, our hypothesizes were tested by using the p-value to weigh the strength of the evidence. The p-value is a number between 0 and 1 and interpreted in the following way: a small p-value $(\leq 0.05)$ indicates strong evidence against the null hypothesis, so the null hypothesis is rejected. A large p-value $(>0.05)$ indicates weak evidence against the null hypothesis, the null hypothesis in not rejected. If p-values are very close to the cutoff (0.05), they are considered to be marginal [23]. Accordingly, results in Table VIII show that hypotheses H1, $\mathrm{H} 3, \mathrm{H} 4, \mathrm{H} 5$, and $\mathrm{H} 8$ are supported and have a high level of statistical significance $(\mathrm{p}<0.05)$, while $\mathrm{H} 2, \mathrm{H} 6, \mathrm{H} 7$, and $\mathrm{H} 9$ are not supported $(\mathrm{p}>0.05)$. For additional analysis, we found that there is no significant correlation between Age and Behavioral Intention $(r=-0.043, \mathrm{p}=0.560, \mathrm{p}>0.05)$.

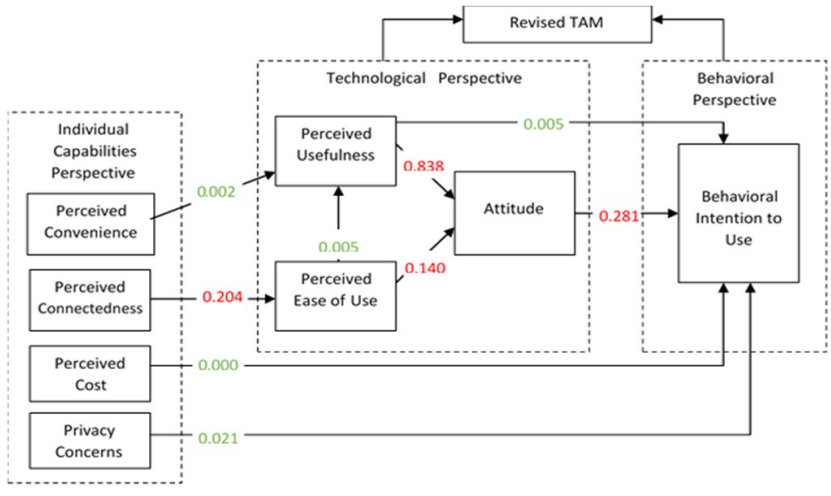

Fig. 2. Summary of our proposed model

TABLE VII. DISCRIMINANT VALIDITY

\begin{tabular}{|c|c|c|c|c|c|c|c|c|}
\hline & PCV & PCO & PC & PCO & ATT & BI & PEO & PU \\
\hline Perceived Convenience (PCV) & 0.837268 & & & & & & & \\
\hline Percieved Connectedness (PCO) & 0.01145 & 0.832103 & & & & & & \\
\hline Percieved Cost (PC) & 0.03312 & 0.04796 & 0.74895 & & & & & \\
\hline Privacy Concerns (PPR) & 0.03168 & 0.03764 & 0.70560 & 0.846573 & & & & \\
\hline $\begin{array}{c}\text { Attitude (ATT) } \\
\end{array}$ & 0.00208 & 0.00043 & 0.00488 & 0.01000 & 0.919761 & & & \\
\hline Behavioral Intention to Use (BI) & 0.00725 & 0.02103 & 0.01061 & 0.01416 & 0.00090 & 0.85708 & & \\
\hline Perceived Ease of Use (PEO) & 0.00001 & 0.00299 & 0.01277 & 0.00800 & 0.01877 & 0.00811 & 0.73291 & \\
\hline Percieved Usefulness (PU) & 0.01369 & 0.00531 & 0.00341 & 0.00152 & 0.01416 & 0.00543 & 0.01020 & 0.82853 \\
\hline
\end{tabular}

TABLE VIII. HYPOTHESIS TESTING

\begin{tabular}{|l|ccc|c|c|}
\hline \multicolumn{3}{|c|}{ Hypothesis } & p & Status \\
\hline H1 & Perceived Convenience & $\rightarrow$ & Perceived Usefulness & 0.002 & Supported \\
\hline H2 & Perceived Connectedness & $\rightarrow$ & Perceived Ease of Use & 0.204 & Not supported \\
\hline H3 & Perceived Cost & $\rightarrow$ & Behavioral Intention to Use & 0.000 & Supported \\
\hline H4 & Privacy Concerns & $\rightarrow$ & Behavioral Intention to Use & 0.021 & Supported \\
\hline H5 & Perceived Ease of Use & $\rightarrow$ & Perceived Usefulness & 0.005 & Supported \\
\hline H6 & Perceived Usefulness & $\rightarrow$ & Attitude & 0.838 & Not supported \\
\hline H7 & Perceived Ease of Use & $\rightarrow$ & Attitude & 0.140 & Not supported \\
\hline H8 & Perceived Usefulness & $\rightarrow$ & Behavioral Intention to Use & 0.005 & Supported \\
\hline H9 & Attitude & $\rightarrow$ & Behavioral Intention to Use & 0.281 & Not supported \\
\hline
\end{tabular}

\section{DISCUSSION}

This study applied an extension to TAM to determine the factors that influence the acceptance of IoT technology for healthcare in KSA. Empirical research was performed to test the study's hypotheses. Most of our findings are in line with findings from previous studies that applied TAM in healthcare systems and e-health. The results offer various useful insights into the acceptance behavior on the IoT technology for healthcare. We found that cost and privacy concerns are the most significant predictors of behavioral intention to use. Moreover, results reveal that perceived usefulness is another important construct that affects the system usage, which in turn is strongly influenced by both perceived convenience and perceived ease of use. On the other hand, results show that perceived connectedness does not affect behavioral intention, neither directly nor indirectly. However, perceived ease of use affects it indirectly through perceived usefulness. Authors in [24-27] found that perceived usefulness and perceived ease of use significantly influence behavioral intention, which is consistent with our results. This is due to the level of effort that goes into learning a new technology or service, which may far outweigh the perceived benefit of the proposed system for many people. Authors in [28-29] found that perceived usefulness was a significant indicator, however, they contradict our findings in which perceived ease of use was an insignificant predictor. This is because their study targeted patients with chronic conditions who tend to rely a lot on the diagnosis and advice (e.g. remote patient monitoring devices) from experts to facilitate disease management and reduce 
medical costs. Authors [30-32] revealed that perceived cost and privacy concerns have a significant impact on forming users' intention. These findings are consistent with ours that show perceived cost as the most significant predictor affecting the behavioral intention. This implies that high cost of implementing this technology can impact the use of IoT services. Thus, inventors must consider cost when implementing this technology for healthcare purposes. Moreover, privacy concerns is another important construct that affects the entire system usage, as patients are extremely concerned and sensitive about the privacy and security of the collected data. This important factor must be kept in mind by healthcare providers as they must be able to provide a technique to support customized data accessing. The results obtained in [26] are quite different from our findings. Specifically, the authors in [26] found that perceived cost was not significantly associated with intention to use mHealth. This variation can be attributed to the availability of mobile services to the population studied in that research. Authors in [33-34] found that perceived convenience has significant impact on forming behavioral intention, which is compatible with our findings. We attribute this to the fact that IoT and the context of providing health support is a relatively new idea, while the underlying technologies are still evolving. Consequently, patients are willing to proceed with this technology and services with little effort or difficulty.

Perceived connectedness has not been extensively considered in the literature. The effect of this construct was found to be non-significant in our study. This observation is in contrast with the findings of other researchers, where connectedness plays a significant role in determining the intention to use a system [35]. This means that patients do not bother about the devices' connectedness. Consequently, patients perceive this technology to be immature and in an early developmental state. Ultimately, although many researchers have considered healthcare services [37, 38] and the use of TAM in their studies [39], we believe that our study added more variables to the original TAM model that could provide a better explanation of patients' acceptance of IoT technology.

\section{CONCLUSION AND FUTURE WORK}

This study assesses empirically the intention of Saudi patients to use IoT technology from a healthcare perspective. To this end, an extended model based upon the TAM was built with eight factors. The proposed model was validated using a instrument designed specifically for this research. In order to ensure that our model can be generalized, the survey was distributed to more than 450 participants, and the responses of 407 of them were considered valid and were further analyzed. SPSS v25.0 and AMOS v23.0 have been utilized to process the results, test the proposed model and the corresponding hypotheses. All test results met normality and validity requirements. The discriminant validity was also tested between factors, showing the sufficiency of our model. The pvalue was used to determine the significance during the hypotheses testing. Results showed that perceived usefulness, cost, and privacy concerns were the leading predictors of behavioral intention to use. Moreover, the perceived usefulness was strongly affected by both perceived convenience and perceived ease of use. However, attitude and perceived connectedness did not have any effect on behavioral intention, directly or indirectly. This research provides the groundwork to explore the process of the actual adoption of IoT services for healthcare. However, as future work, more factors should be identified and added to the model, in order to gain more insights and ensure greater success of such service. Moreover, the model could be extended to include a larger number of patients. In addition, we can extend our current findings by investigating the moderating effects of age, cultural background, and other factors.

\section{REFERENCES}

[1] R. Minerva, A. Biru, D. Rotondi, Towards a Definition of the Internet of Things (IoT), IEEE Internet Initiative, 2015

[2] A. Al-Fuqaha, M. Guizani, M. Mohammadi, M. Aledhari, M. Ayyash, "Internet of things: a survey on enabling technologies, protocols, and applications", IEEE Communications Surveys \& Tutorials, Vol. 17, No. 4, pp. 2347-2376, 2015

[3] C. A. da Costa, C. F. Pasluosta, B. Eskofier, D. B. da Silva, R. da Rosa Righi, "Internet of health things: toward intelligent vital signs monitoring in hospital wards", Artificial Intelligence in Medicine, Vol. 89, pp. 61-69, 2018

[4] M. Ersue, D. Romascanu, J. Schonwalder, A. Sehgal, Management of Networks with Constrained Devices: Use Cases, RFC 7548, available at: https://tools.ietf.org/html/rfc7548, 2015

[5] A. Dillon, M. Morris, "User acceptance of new information technology: Theories and models", Annual Review of Information Science and Technology, Vol. 14, No. 4, pp. 3-33, 1996

[6] L. Gao, X. Bai, "A unified perspective on the factors influencing consumer acceptance of internet of things technology", Asia Pacific Journal of Marketing and Logistics, Vol. 26, No. 2, pp. 211-231, 2014

[7] F. D. Davis, "Perceived Usefulness, perceived ease of use, and user acceptance of information technology", MIS Quarterly, Vol. 13, No. 3, pp. 319-340, 1989

[8] V. Venkatesh, J. Y. L. Thong, X. Xu, "Unified theory of acceptance and use of technology: A synthesis and the road ahead", Journal of the Association for Information Systems, Vol. 17, No. 5, pp. 328-376, 2016

[9] B. H. Sheppard, J. Hartwick, P. R. Warshaw, "The theory of reasoned action: A meta-analysis of past research with recommendations for modifications and future research", Journal of Consumer Research, Vol. 15, No. 3, pp. 325-343, 1988

[10] S. Y. Yousafzai, G. R. Foxall, J. G. Pallister, "Technology acceptance: a meta-analysis of the TAM: Part 2", Journal of Modelling in Management, Vol. 2, No. 3, pp. 281-304, 2007

[11] R. J. Holden, B. T. Karsh, "The technology acceptance model: its past and its future in health care", Journal of Biomedical Informatics, Vol. 43, No. 1, pp. 159-172, 2010

[12] A. K. Yarbrough, T. B. Smith, "Technology acceptance among physicians: a new take on TAM", Medical Care Research and Review, Vol. 64, No. 6, pp. 650- 672, 2007

[13] V. Venkatesh, H. Bala, "Technology acceptance model 3 and a research agenda on interventions", Decision Sciences, Vol. 39, No. 2, pp. 273315,2008

[14] T. Teo, C. B. Lee, "Attitudes towards computers among students in higher education: A case study in Singapore", British Journal of Educational Technology, Vol. 39, No. 1, pp. 160-162, 2008

[15] T. Teo, "A structural equation modeling of factors influencing student teachers' satisfaction with e-learning", British Journal of Educational Technology, Vol. 41, No. 6, pp. E150-E152, 2010

[16] S. McCoy, A. Everard, B. M. Jones, "An examination of the technology acceptance model in Uruguay and the US: A focus on culture", Journal of Global Information Technology Management, Vol. 8, No. 2, pp. 2745,2005 
[17] M. Srite, E. Karahanna, "The role of espoused national cultural values in technology acceptance", MIS Quarterly, Vol. 30, No. 3, pp. 679-704, 2006

[18] J. Charan, T. Biswas, "How to calculate sample size for different study designs in medical research?", Indian Journal of Psychological Medicine, Vol. 35, No. 2, pp. 121-126, 2013

[19] D. George, SPSS for Windows Step by Step: A Simple Guide and Reference, Pearson Education India, 2011

[20] M. Tenehaus, V. E. Vinzi, Y. M. Chatelin, C. Lauro, "PLS path modeling", Computational Statistics \& Data Analysis, Vol. 48, No. 1, pp. 159-205, 2005

[21] M. Wetzels, G. Odekerken-Schroder, C. Van Oppen, "Using PLS path modeling for assessing hierarchical construct models: Guidelines and empirical illustration", MIS Quarterly, Vol. 33, No. 1, pp. 177-195, 2009

[22] J. F. Hair Jr, G. T. M. Hult, C. Ringle, M. Sarstedt, A Primer on Partial Least Squares Structural Equation Modeling (PLS-SEM), SAGE Publications, 2016

[23] P. H. Westfall, S. S. Young, Resampling-based Multiple Testing: Examples and Methods for p-value Adjustment, John Wiley \& Sons, 1993

[24] Y. Zhao, Q. Ni, R. Zu, "What factors influence the mobile health service adoption? A meta-analysis and the moderating role of age", International Journal of Information Management, Vol. 43, pp. 342-350, 2018

[25] M. Weng. The Acceptance of Wearable Devices for Personal Healthcare in China, Msc Thesis, University of Oulu, 2016

[26] M. I. Cahita, N. A. Hodgson, C. Budhathoki, H. R. Han, "Intention to use mHealth in older adults with heart failure", The Journal of Cardiovascular Nursing, Vol. 32, No. 6, pp. E1-E7, 2017

[27] A. M. AlBar, M. R. Hoque, "Patient acceptance of e-health services in Saudi Arabia: An integrative perspective", Journal of Telemedicine and e-Health, 2018

[28] N. Hossain, F. Yokota, N. Sultana, A. Ahmed, "Factors influencing rural end-users' acceptance of e-health in developing countries: A study on portable health clinic in Bangladesh", Telemedicine and e-Health, Vol. 25, No. 3, pp. 221-229, 2019

[29] N. Sun, P. L. P. Rau, "The acceptance of personal health devices among patients with chronic conditions", International Journal of Medical Informatics, Vol. 84, No. 4, pp. 288-297, 2015

[30] M. R. Hoque, Y. Bao, G. Sorwar, "Investigating factors influencing the adoption of e-health in developing countries: A patient's perspective", Informatics for Health and Social Care, Vol. 42, No. 1, pp. 1-17, 2017

[31] A. J. E. de Veer, J. M. Peeters, A. E. M. Brabers, F. G. Schellevis, J. J. D. J. M. Rademakers, A. L. Francke, "Determinants of the intention to use e-Health by community dwelling older people", BMC Health Services Research, Vol. 15, 2015

[32] D. Pal, S. Funilkul, N. Charoenkitkarn, P. Kanthamanon, "Internet-ofthings and smart homes for elderly healthcare: An end-user perspective", IEEE Access, Vol. 6, pp. 10483-10496, 2018

[33] V. Dutot, F. Bergeron, K. Rozhkova, N. Moreau, "Factors affecting the adoption of connected objects in e-health: A mixed methods approach", Systemes d'Information et Management, Vol. 23, No. 4, 2019

[34] H. Emad, H. M. El-Bakry, A. Asem, "A modified technology acceptance model for health informatics", International Journal of Artificial Intelligence and Mechatronics, Vol. 4, No. 4, pp. 153-161, 2016

[35] S. Dunnebeil, A. Sunyaev, I. Blohm, J. M. Leimeister, H. Krcmar, "Determinants of physicians' technology acceptance for e-health in ambulatory care", International Journal of Medical Informatics, Vol. 81, No. 11, pp. 746-760, 2012

[36] M. Alanazi, B. Soh, "Internet-of-Things for healthcare purposes: extending the technology acceptance model for Saudi Arabia patients", International Journal of Computer Science and Network Security, Vol. 19, No. 2, pp. 74-81, 2019

[37] F. E. Idachaba, E. M. Idachaba, "Robust e-health communication architecture for rural communities in developing countries", Engineering, Technology \& Applied Science Research, Vol. 2, No. 3, pp. $237-240,2012$
[38] D. Virmani, P. Girdhar, P. Jain, P. Bamdev, "FDREnet: face detection and recognition pipeline", Engineering, Technology \& Applied Science Research, Vol. 9, No. 2, pp. 3933-3938, 2019

[39] P. Shayan, E. Iscioglu, “An assessment of students' satisfaction level from learning management systems: case study of Payamnoor and Farhangian Universities", Engineering, Technology \& Applied Science Research, Vol. 7, No. 4, pp. 1874-1878, 2017

\section{AUTHORS PROFILE}

Meshari Alanazi received his BSc degree in Computer Science from Northern Border University and his MSc degree in Computer Science from Western Illinois University in USA 2016. During 2016-2018, he was a Lecturer in Northern Border University, and in 2018 he started his $\mathrm{PhD}$. in La Trobe University.

Ben Soh obtained his $\mathrm{PhD}$ in Computer Science \& Engineering from La Trobe University in 1995. Since then, he has successfully supervised to completion nine $\mathrm{PhD}$ students and published more than 160 peer-reviewed research papers. He has made significant contributions in the following research areas: Fault-Tolerant and Secure Computing, Cloud Computing, Information Systems Research, Pervasive Wireless Network Communications, and Business Process Management. 